\title{
Connectivity considerations for mission planning of a search and rescue drone team
}

\author{
Evşen YANMAZ ADAM* (D) \\ Department of Electrical and Electronics Engineering, Faculty of Engineering, \\ Özyeğin University, İstanbul, Turkey
}

$\begin{array}{lllll}\text { Received: } 06.12 .2019 & \text { - } & \text { Accepted/Published Online: 21.02.2020 } & \text { Final Version: } 29.07 .2020\end{array}$

\begin{abstract}
In this paper, we analyze the mission success performance and mission times of centralized, distributed, and hybrid path-planning methods for a drone team whose mission is to find a target and inform the ground control. We propose two methods that integrate connectivity into the search mission path decisions. We observe that even though the coverage path-planning leads to lower search times, when target connectivity is also required, schemes that incorporate end-end connectivity into path planning result in at least $50 \%$ better mission times for small communication ranges and lower number of drones. Our results also indicate that methods to efficiently allocate resources to search and communication tasks in mission-oriented drone networks need to be designed.
\end{abstract}

Key words: Drones, unmanned aerial vehicles, path planning, target detection, monitoring, swarms, networking

\section{Introduction}

Drone network applications have grown significantly in the past decade. Drones are utilized as sensors, for network provisioning, and for delivery among others [1]. While most drone or unmanned aerial vehicle (UAV) use cases demand area coverage [2], it has been shown that some form of connectivity to a single entity (e.g., control center or base station) or multiple (e.g., mobile users and UAV swarm members) entities is also desirable. Paths for UAVs deployed as part of the wireless network either to assist in disasters or to meet changing traffic demands need to be planned according to the network conditions and available resources (in terms of power and bandwidth) [3]. However, demand for connectivity can also appear in applications other than UAV-assisted wireless networks. Connectivity can be event-driven as in search and rescue or delivery systems, periodic as in remote sensing or environmental monitoring, or continuous as in surveillance systems. Furthermore, for fully autonomous multi-UAV system operation, drone-to-drone communication will be highly necessary.

In the present paper, we consider a team of UAVs deployed for searching an area for a single target, where the mission is considered successful once the target is found and this information is delivered to the ground control. Cooperative search with UAVs has been considered from several viewpoints in the literature including search time, detection accuracy, and energy consumption. In the following, we focus on related work from the communications and networking viewpoint for coverage/target detection applications. Sometimes $[4,5]$ bandwidth limitations are not considered in the path plan, whereas connectivity is considered as a constraint $[6,7]$ or as a mission goal $[8,9]$. The connectivity problem can be solved either by incorporating communication needs into the UAV path-planning problem [10-14] or additional UAVs can be deployed as communication relays

*Correspondence: eyanmaz@alumni.cmu.edu 
to provide connectivity to the searching UAVs [15]. These works clearly illustrate the diversity of communication needs in target detection applications and the trade-off between coverage and connectivity very similar to the earlier works on wireless sensor networks. Furthermore, the problem of search and connectivity optimization can be addressed using different solution techniques including potential fields, mixed integer linear programming, space-filling curves, particle swarm optimization, and genetic algorithm (GA) [10]. Previously [2], we have shown that the communication needs in a UAV team depend on the application. In the present paper, we focus on a search and rescue application and in contrast to previous works our main goal is to illustrate the connectivity limitations in a variety of search (coverage) optimum path planners as well as analyzing the impact of integrating connectivity constraints into the planning process on the mission performance of a UAV team. We provide a general analysis that can be utilized in determining when to consider connectivity and in tuning algorithm parameters according to the resources (for instance number of drones) at hand. Assuming a team of drones (with onboard sensing, embedded processing, and wireless network interface), the aim of the present work is to analyze the mission success performance of a drone team that flies paths with or without connectivity considerations. To this end, we first introduce representative centralized and distributed path planning schemes that consider only area coverage or multihop connectivity to the BS. By selecting representative schemes we enable generalization of the findings of our analysis to other planners. For instance, while we use a search optimum algorithm that utilizes a GA, the mission performance analysis is not bound to the optimization method but the optimization outcome. Furthermore, the distributed and centralized approaches have some fundamental differences in their level of prior knowledge, adaptability, robustness to mission changes, complexity, and autonomy and their analysis provides insight into achievable mission outcomes for a range of path-planning algorithms. We then propose two novel path-planning schemes that incorporate multihop connectivity into the coverage (search) path-planning decisions. We analyze the performance of the UAV team in terms of mission success and time including both target detection and BS notification. Our main contributions can be summarized as follows:

- Two novel multi-UAV path planners that integrate connectivity limitations into coverage (search) pathplanning algorithms

- Comparison of distributed and centralized path planners in terms of mission success and mission times for ideal and nonideal sensing and channel conditions

- Connectivity analysis of path planners during a search and rescue mission

While we use simulations to conduct our analysis, we utilize real-world measurement outcomes from the literature as much as possible when we determine the system parameter values and use cases [1]. Implementation of our methods on a multi-UAV testbed is a future focus point to determine the impact of real-world limitations. Our analysis shows that as the number of drones increases, network connectivity improves significantly, allowing coverage-based schemes to have better success probabilities. However, under stringent connectivity conditions, i.e. if the communication range and number of drones are low, connectivity-based distributed schemes outperform the coverage-aware centralized and distributed schemes by at least $50 \%$ by enabling notification as soon as the target is detected. The proposed hybrid schemes overcome the limitations of path planning that consider only coverage or connectivity under certain conditions. We further analyze the immediate notification probability and the percentage of time that a target would be connected to the BS over the drone network. We illustrate that while connectivity-based schemes may be more suitable for missions that require continuous connectivity, coverage path planners might require notification delay tolerance. Our results indicate that methods to efficiently allocate resources to search and communication tasks in drone networks need to be designed. 
The remainder of the paper is organized as follows. In Section 2, we provide the system assumptions and performance metric definitions. The multi-UAV planners under investigation are presented in Section 3 . The results are given in Section 4 and the paper is concluded in Section 5.

\section{System model}

\subsection{Assumptions}

Here we provide assumptions common to the path-planning schemes in the present paper (system parameters are summarized in Table 1). The UAVs take off from the ground BS together to search for a single target that occurs at a random location $\left(x_{t}, y_{t}\right)$ within the search region and lasts for a duration of $t_{d}$ s. Once the target is found, the ground personnel need to be notified. The search region, $A$, is a square area with sides of length $a$. The UAV team consists of $N_{m}$ drones, which fly with speeds equal to $V_{m}$ at the same altitude $(h)$. In a practical system, the speed or more generally motion dynamics of the drones can affect the mission times, sensing quality, wireless channel quality, etc. For a fair comparison between the path planning methods and a better understanding of how connectivity factors into mission success, we consider constant speeds in the present paper. We assume that each drone has a fixed communication range of $r_{c}$, following distance-based large-scale fading verified by our earlier measurements [1]. Several channel models have been considered for UAV communications ([16] and references therein). The main characteristic of the UAV channel from measurement campaigns is that it undergoes distance-based path loss and has a strong line-of-sight component, which leads to Rician or more generally Nakagami fading. The exact distribution depends on the type of the drones, the onboard transceiver, the antenna considerations, motion dynamics, the terrain, etc. In the present work, we only require that there is a multihop link to the BS on average and the exact channel distribution, and hence the instantaneous quality of the links and the end-to-end throughput is outside the scope of our study. However, to illustrate the impact of the environment on the mission, we also provide a brief connectivity analysis with log-normal shadowing corresponding to an urban search and rescue scenario with parameters taken from realworld air-to-ground channel measurements [17]. We further assume that the drones know their own positions (e.g., from onboard global positioning system (GPS)) and are equipped with sensors (e.g., cameras) to detect the target. We assume that the drones rely on GPS to avoid collisions and consider the GPS update rate and accuracy while determining the sensing period and direction change times for the distributed schemes (these two parameters determine how closely the drones can fly). Further sense-and-avoid techniques are not considered. We use a simplified sensing model. We assume that the UAVs have a fixed sensing range of $r$ and as soon as the target is within the sensing coverage it can be detected without error. This binary method of detection, though simplified, is sufficient to analyze the performance trends of the path planning strategies. The impact of sensor imperfections and more realistic sensing models have been heavily analyzed and integrated into coordinated searches in earlier works $[5,18]$. We will briefly discuss the implications of considering error-free sensing in Section 3. We will also illustrate the impact of imperfect sensing on the target detection performance of the UAV team in Section 4.

\subsection{Definitions}

The UAV mission is defined as finding a target in a search area and notifying the ground personnel in a given time. Mission success probability $P_{s}$ is given by

$$
P_{s}=\operatorname{Pr}\left\{\mathrm{BS} \text { notified within } t_{d}\right\}
$$


The mission depends on both the coverage and connectivity of the UAV team. Coverage relates to target search and detection and a target is detected if it is sensed by at least one UAV within target duration. For a given time $t$ and UAV position set of $X Y_{u}^{t}=\left\{\left(x_{i}(t), y_{i}(t)\right): i=1,2, \ldots, N_{m}\right\}$, the probability of detection is given by

$$
P_{d}= \begin{cases}1, & \text { if } \min \left\{d_{i}^{t}\right\} \leq r \text { and } t \leq t_{d} \\ 0, & \text { otherwise }\end{cases}
$$

where $d_{i}^{t}$ is the Euclidean distance between the target and the UAV $i, i=1,2, \ldots, N_{m}$ at time $t$ :

$$
d_{i}^{t}=\sqrt{\left(x_{i}(t)-x_{t}(t)\right)^{2}+\left(y_{i}(t)-y_{t}(t)\right)^{2}}
$$

Once a target is detected, the position of the target needs to be propagated to the BS. We consider multihop connectivity, where two nodes are connected as soon as they are within each other's communication range $r_{c}$. UAVs forward the information about the target to their neighbors at the time of contact. At a given time $t$, the UAV network can be specified as a graph $G_{t}=\left(N, E_{t}\right)$, where $N$ is the set of UAVs and the BS (i.e. $|N|=N_{m}+1$ ) and $E_{t}$ is the wireless links satisfying

$$
E_{t}:=\left\{\operatorname{edge}(j, i) \text { if and only if } d_{i j}(t) \leq r_{c}, \forall i, j \in N\right\}
$$

where $d_{i j}(t)$ is the Euclidean distance between nodes $i$ and $j$ in set $N$. Without loss of generality, we set node 1 to be the BS. The corresponding adjacency matrix $A_{t}$ is defined as $A_{t}=\left(a_{j i}(t)\right), 1 \leq j, i \leq N_{m}+1$, where

$$
a_{j i}(t)= \begin{cases}1, & \text { if }(j, i) \in E_{t} \\ 0, & \text { otherwise }\end{cases}
$$

Let us denote the set of UAVs with knowledge of the target location by $R$. The BS can be notified if and only if there exists a path in the graph $G_{t}$ between any UAV in $R$ and the BS at any time $t \leq t_{d}$. To find the probability of notification and hence mission success probability, we do a depth-first search over the adjacency matrix $A_{t}$ to determine whether a path exists between the nodes in $R$ and the BS at any time $t \leq t_{d}$. We also analyze the probability of simultaneous detection of a target and BS notification to understand the portion of time spent for establishing connectivity. Finally, we define target reachability as the percentage of time the target has a multihop connection to the BS over the drone network. Although our mission is considered a success once the BS is notified of the target location, target reachability can be relevant to other applications such as monitoring or surveillance. The reachability performance presented in the present paper can serve as a benchmark for designing path-planning algorithms that aim, for instance, to track targets.

\section{Multi-UAV path planning}

In the following, we first describe multi-UAV path-planning algorithms that either focus on fast area coverage or multihop connectivity to the BS. Then we propose two algorithms that consider both coverage and connectivity in the planned paths. For the centralized algorithms, the paths are generated at the BS prior to the mission, utilizing full knowledge of the search area and the UAV team. For the distributed algorithms, the paths are generated during flight based on local information. More specifically, each UAV periodically senses its neighbors and decides if a direction change is necessary, depending on the relative positions of the UAVs in its vicinity. The algorithms return the next direction $\left(\theta^{+}\right)$during the mission at the end of the sensing period. If, at the 
YANMAZ ADAM/Turk J Elec Eng \& Comp Sci

Table 1. System parameters.

\begin{tabular}{|l|l|}
\hline Parameter & Description \\
\hline$A$ & Square search area $(a \times a)$ \\
\hline$N_{m}, V_{m}, h$ & Number, velocity, and altitude of UAVs \\
\hline$r_{c}$ & Communication range of a UAV \\
\hline$r$ & Sensing range of a UAV \\
\hline$t_{d}$ & Target (event) duration \\
\hline$t_{s}$ & Sensing period \\
\hline
\end{tabular}

time of direction change, a UAV does not have any neighbors, the direction is not changed. Note that the sensing period is a design parameter and depends on the system parameters such as $N_{m}$ and $r$. The same algorithm is run on all UAVs. If a UAV estimates that it would leave the search area at the end of the sensing period, it chooses a random direction toward the search area before the period is over. A qualitative comparison of the benchmark path-planning algorithms is given in Table 2. An in depth theoretical analysis of centralized and distributed cooperative UAV planning algorithms under investigation is conducted in Khan et al. [18] and Yanmaz et al. [19], respectively.

Table 2. Comparison of path-planning methods.

\begin{tabular}{|l|l|l|l|}
\hline Property & $\begin{array}{l}\text { Multiple traveling salesmen } \\
\text { problem (mTSP) }\end{array}$ & $\begin{array}{l}\text { Coverage-based } \\
\text { (CovB) }\end{array}$ & $\begin{array}{l}\text { Connectivity-based } \\
\text { (ConB) }\end{array}$ \\
\hline Online & No & Yes & Yes \\
\hline Centralized & Yes & No & No \\
\hline Prior knowledge & Yes & No & No \\
\hline $\begin{array}{l}\text { Node failures/ } \\
\text { additions }\end{array}$ & Needs to replan & Robust & Robust \\
\hline Coverage & Guaranteed & In probability & N/A \\
\hline Connectivity & N/A & N/A & In probability \\
\hline Onboard processing & Not required & $\begin{array}{l}\text { Detection, direction } \\
\text { decision }\end{array}$ & $\begin{array}{l}\text { Detection, route } \\
\text { discovery, direction } \\
\text { decision }\end{array}$ \\
\hline Suitable for & $\begin{array}{l}\text { Delivery, environmental } \\
\text { monitoring, infrastructure } \\
\text { inspection, search, etc. }\end{array}$ & $\begin{array}{l}\text { Crop spraying, } \\
\text { environmental } \\
\text { monitoring, search, } \\
\text { mapping, etc. }\end{array}$ & $\begin{array}{l}\text { Surveillance, network } \\
\text { provisioning, event } \\
\text { streaming, etc. }\end{array}$ \\
\hline
\end{tabular}

\subsection{Coverage-aware path planning}

Coverage refers to the area search phase of the mission and the goal of the coverage-aware path-planning schemes is to minimize search times and/or guarantee full area-coverage within a given time. We assume perfect sensing in the present paper. However, in a practical system where sensing is of probabilistic nature and there are sensor errors, the centralized schemes have a likelihood of missing a target since there is no redundancy (overlap) in the drone paths. Methods have been proposed to improve the search performance for such schemes including 
belief map merging, repetitive sensing, multiple tours, etc. [5, 18]. Distributed path-planning schemes on the other hand have an inherent redundancy. Furthermore, they can be more easily extended to include sensing quality in the decision making process by adding their observations (sensor readings) to the already exchanged local information to determine their paths.

\subsubsection{Path planning with the multiple traveling salesmen Problem}

UAV path planning can be modeled as a multiple traveling salesmen problem (mTSP), where the cities to be visited are determined by dividing the search region $A$ into $(a / r)^{2}$ equal-sized, disjoint cells. The objective of the mTSP is to determine a set of routes for $N_{m}$ drones such that the total traveled distance is minimized. The routes should start and end at the BS and each cell must be visited exactly once by only one drone. Note that mTSP is an NP-hard problem and can be solved using several methods including dynamic programming, ant colony optimization, and particle swarm optimization to name a few [20]. Each approach has different time and space complexity. Depending on the computation limitations at the BS of the practical system to be deployed, any of these algorithms can be used. Since in the present paper we are interested in the connectivity analysis of the resulting paths, we do not have any presumptions regarding computation time and we set up a GA for the mTSP path planner [21]. The algorithm takes the cell locations, number of $\operatorname{UAVs}\left(N_{m}\right)$, minimum tour length for each UAV, population size, and number of iterations as input and computes the paths to be traveled prior to flight. The mTSP planner does not consider any connectivity constraints but aims only to minimize the cooperative search time. However, by generating paths that lead back to the home base, we enable notification when the UAVs fly back at the latest.

\subsubsection{Coverage-based path planner}

An alternative to the centralized mTSP planner that has complete knowledge about the search area is the distributed coverage-based (CovB) path planner, which utilizes artificial potential fields (APFs) [22]. Only the boundaries of the search area are known by the UAVs prior to the mission. The CovB planner aims to minimize the overlap between sensed areas by the individual members of the UAV team, by allowing neighboring UAVs to "push" each other with forces inversely proportional to the distance between them.

We present the forces on a given UAV in nonboundary areas next. For details of the algorithm readers are referred to Yanmaz and Guclu [22]. We use $\widehat{\theta}$ notation for the unit vector with direction $\theta$. The resultant force applied on a given UAV $i$ with current direction $\theta_{i}^{c}$ and a sensing neighbor set of $N_{i}^{s}$ at the time of direction change is

$$
\begin{aligned}
\overrightarrow{R_{v}^{c o v}} & =\vec{F}_{i i}+\sum_{j \in N_{i}^{s}} \vec{F}_{i j}, \text { where } \\
\overrightarrow{F_{i i}} & =\frac{1}{r} \widehat{\hat{\theta}_{i}^{c}} \text { and } \\
\overrightarrow{F_{i j}} & =\frac{1}{d_{i j}} \widehat{\theta_{i j}} \text { and } \theta_{i j}=\tan ^{-1}\left(\frac{y_{j}-y_{i}}{x_{j}-x_{i}}\right)
\end{aligned}
$$

For convenience, time index is dropped from the UAV positions $\left(x_{i}(t), y_{i}(t)\right)$ and the distance between UAVs $i$ and $j, d_{i j}(t)$. The next direction $\theta_{i}^{+}$is the angle of the resultant force $\overrightarrow{R_{v}^{c o v}}$ given in Eq. (6). 


\subsection{Connectivity-based path planner}

The connectivity-based (ConB) path-planning algorithm is also distributed and utilizes APFs [9]. In addition to the current positions and directions of neighboring UAVs, the resulting network graph and connectivity are also utilized in the decision-making process. In particular, in nonboundary areas, within every sensing period the connectivity of a given UAV $i$ to the BS as well as its neighbors is checked at incremental time intervals utilizing adjacency matrices (Eq. (5)) and depth-first search. If at any time within the sensing period the UAV $i$ would lose its connectivity to the BS, direction change would be initiated. If no disconnections are predicted, then the direction change is done at the end of the sensing period as usual. Using the same conventions as above, the resultant force applied on a UAV $i$ for the ConB algorithm is

$$
\begin{aligned}
\overrightarrow{R_{v}^{c o n}} & =\vec{F}_{i i}+\vec{F}_{i j}, \text { where } \\
\vec{F}_{i i} & =\widehat{\theta_{i}^{c}} \text { and } \\
\vec{F}_{i j} & =\widehat{\theta_{i j}} \text { and } \theta_{i j}=\tan ^{-1}\left(\frac{y_{j}^{\text {contact }}-y_{i}^{+}}{x_{j}^{\text {contact }}-x_{i}^{+}}\right)
\end{aligned}
$$

In Eqs. (9) and (11), $j$ represents the neighbor of UAV $i$ that would have the longest contact duration,

if both UAVs kept their current headings and $\left(x_{j}^{\text {contact }}, y_{j}^{\text {contact }}\right)$ is the location where UAVs $i$ and $j$ would lose contact. The goal is simultaneously avoiding too many direction changes and isolated UAVs in the network. The position of node $i$ at the end of next time increment is denoted by $\left(x_{i}^{+}, y_{i}^{+}\right)$. The next direction $\theta_{i}^{+}$is the angle of the resultant force $R_{v}^{\overrightarrow{c o n}}$ given in Eq. (9).

\subsection{Joint connectivity and coverage-aware path planning}

In the following, we propose two path planners that utilize both coverage and connectivity in the decision-making process: (i) mTSP-ConB and (ii) ConCov.

mTSP-ConB is a hybrid of the mTSP and ConB algorithms (see Algorithm 1). It reserves $N_{r}$ drones only for searching and the remaining $N_{m}-N_{r}$ nodes assist in providing connectivity. To this end, $N_{r}$ drones follow mTSP paths that are generated before flight, whereas the remaining drones update their positions according to ConB during flight. $N_{r}$ can be chosen such that the search is completed within a given time. The $N_{m}-N_{r}$ drones, while redundant for search purposes, help achieve better resource utilization for the overall mission goal, which also includes BS notification. The ConCov algorithm, on the other hand, is fully distributed and combines the ConB and CovB algorithms (see Algorithm 2). In particular, ConCov gives a weight, $\alpha$, to search and notification goals of the mission. To this end, the resultant force applied to each UAV is

$$
\overrightarrow{R_{v}}=\alpha \frac{\overrightarrow{R_{v}^{c o v}}}{\left|\overrightarrow{R_{v}^{c o v}}\right|}+(1-\alpha) \frac{\overrightarrow{R_{v}^{c o n}}}{\left|\overrightarrow{R_{v}^{c o n}}\right|}
$$

where $R_{v}^{c o v}$ and $R_{v}^{c o n}$ are given in Eqs. (6) and (9), respectively. The resultant force combines the "repellant" force of CovB against coverage overlaps with the "attraction" force of ConB against disconnections. The next direction $\theta_{i}^{+}$in this case is the angle of the resultant force $\overrightarrow{R_{v}}$. Choice of $\alpha$ depends on how much weight is to be given on connectivity. As $\alpha$ goes to zero (one), ConCov approaches ConB (CovB).

Both hybrid schemes have advantages regarding notification performance over their native mTSP and CovB at the cost of search time. However, since the mission involves both search and notification, the mission 


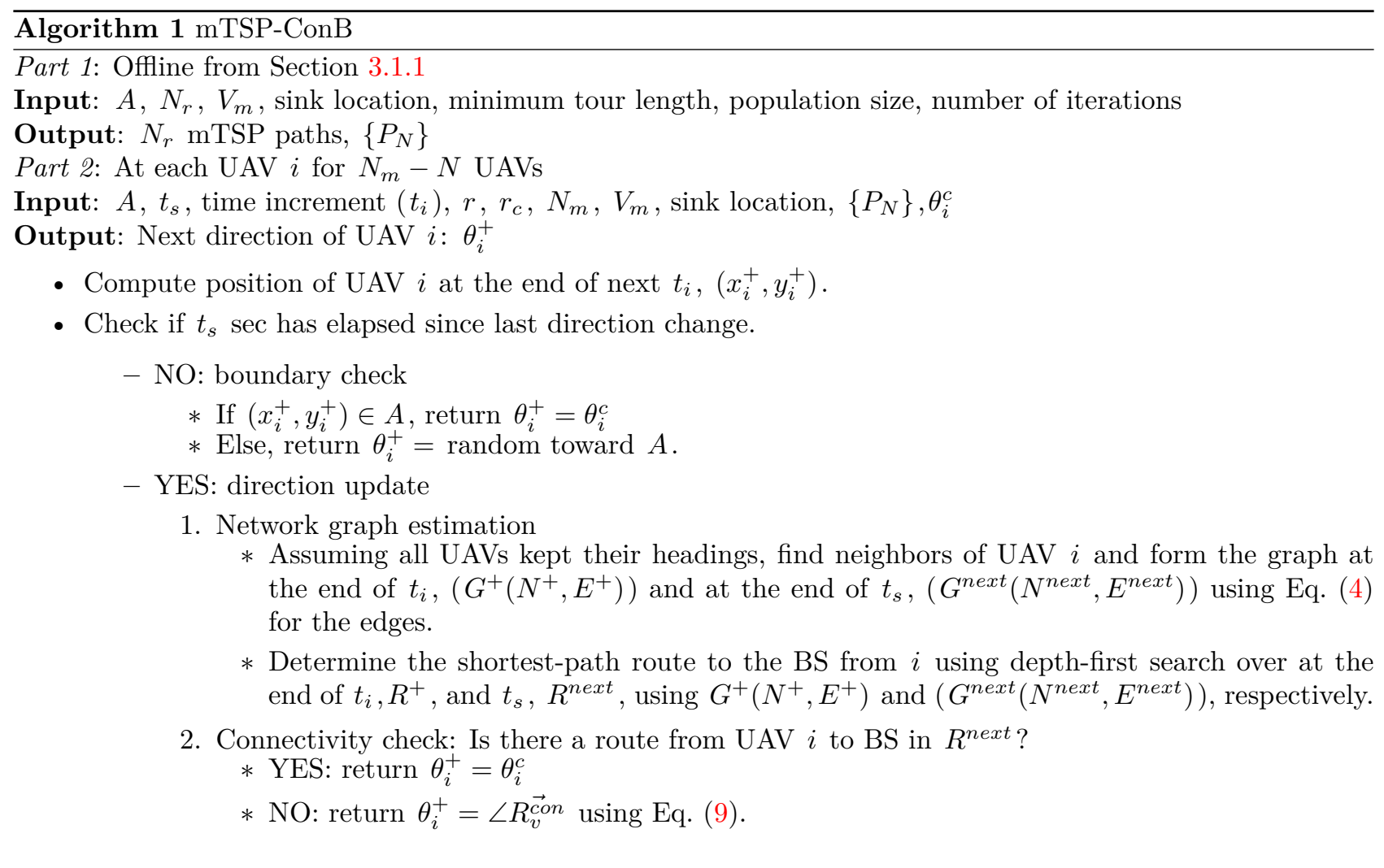

success probability can also be improved depending on the choice of the design parameters $N_{r}$ and $\alpha$. Our future work focuses on determining the parameter values for optimized mission success probability. In addition to the mission performance, the hybrid algorithms also have some indications regarding computation times. For a given $N_{m}$, the mTSP-ConB scheme will gain in computation time due to fewer salesmen $\left(N_{r}\right)$ in the offline phase, but suffer due to the introduction of depth-first search in the ConB component. Similarly, ConCov will suffer over CovB due to depth-first search. An advantage of mTSP-ConB is that it guarantees coverage at a given time, which ConCov can guarantee only in probability. However, the fully distributed nature of ConCov results in a more robust system against node failures or environment changes.

\section{Results and discussion}

\subsection{Simulation setup}

In this section, we compare the performances of mTSP and APF-based UAV path-planning strategies via Monte Carlo simulations over 2000 runs. For the target detection performances of CovB and ConB schemes readers are referred to Yanmaz [23]. The simulation parameters are given in Table 3. For the GA ${ }^{1}$ of mTSP and mTSP-Con, the population size is 80 and number of iterations is 5000. For the given $r$ and $a$, the number of grid points for mTSP such that the search area is fully covered is 64 . The area size and the velocity are chosen taking into account a maximum flight time of $1 \mathrm{~h}$. The region and the velocity can be scaled down or up depending on the flight time of the deployed drones. The communication range $r_{c}$ is determined using theoretical free-space path loss over air-ground links and mapping different levels of desired throughput rates (i.e. high, medium,

\footnotetext{
${ }^{1}$ We use Joseph Kirk's implementation in MATLAB.
} 


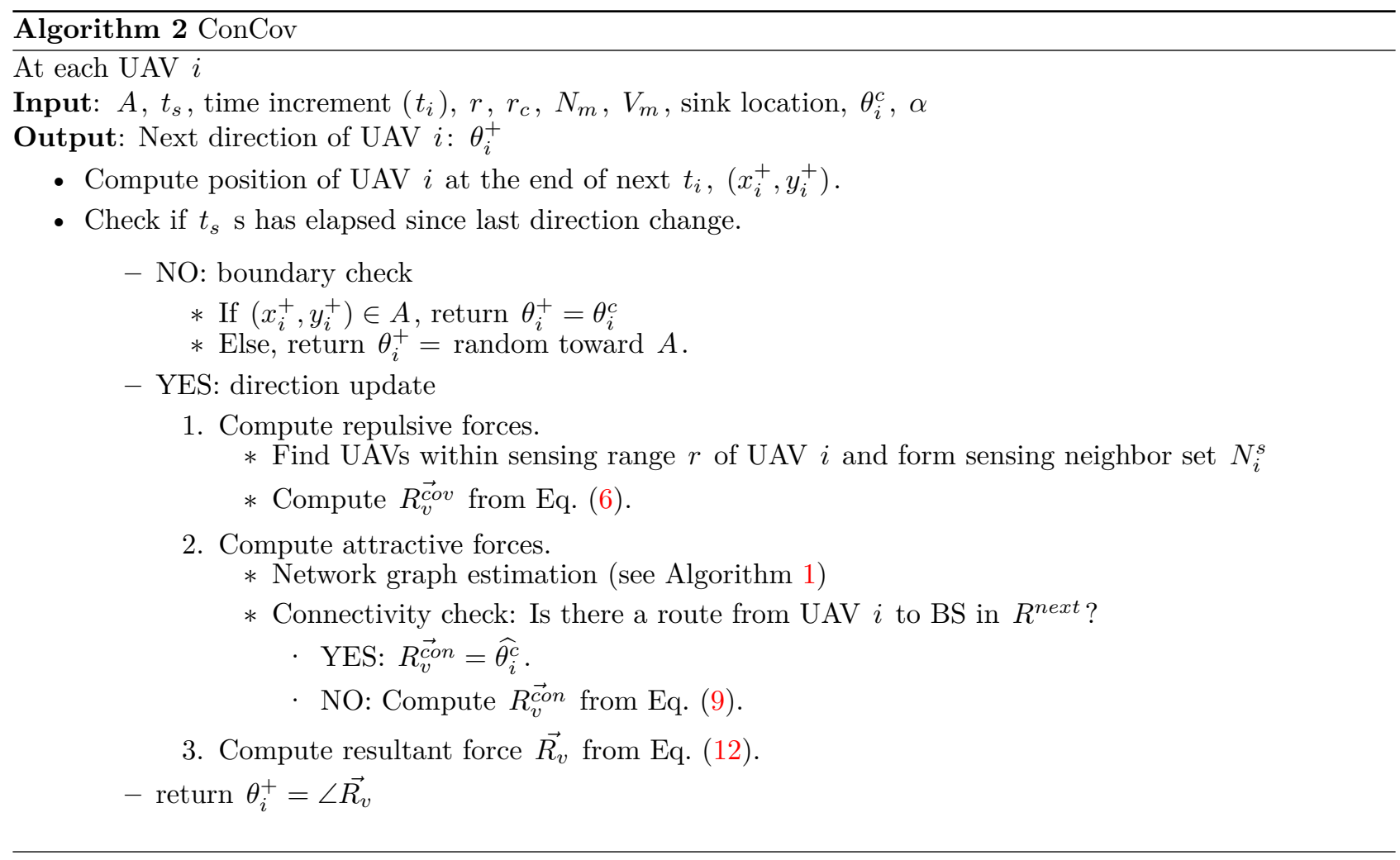

low) to short, medium, and long distances. The sensing period is chosen in accordance with the expected GPS position update period on real vehicles. The maximum flight time is computed according to the full-coverage path generated by mTSP for 2 UAVs and serves only as a reference limit. For the ConCov algorithm, we set $\alpha$ to 0.5 and give equal weight to coverage and connectivity. For the mTSP-Con algorithm, we set $N_{r}$ for the offline phase to 6 . We selected this value according to the search performance of native mTSP for the given number of cities and the flight times of practical drones. We study a single stationary target scenario, where the target appears at a random location within the search area and lasts for a duration of $t_{d} \mathrm{~s} . N_{m}$ drones are deployed for the mission. Assuming a target is detected without error as soon as it is within sensing range of a UAV, the performance metrics of interest are: i) probability of mission success (i.e. detection and notification within $\left.t_{d}\right)$; ii) mission phase times; iii) target reachability.

Table 3. Simulation parameters.

\begin{tabular}{|l|l|}
\hline Parameter & Value \\
\hline$a$ & $4000 \mathrm{~m}$ \\
\hline$V_{m}$ & $5 \mathrm{~m} / \mathrm{s}$ \\
\hline$r$ & $500 \mathrm{~m}$ \\
\hline Sensing period & $2 \mathrm{~s}$ \\
\hline Maximum flight time & $4000 \mathrm{~s}$ \\
\hline$r_{c}$ & $\{500,1000,2000\} \mathrm{m}$ \\
\hline
\end{tabular}




\subsection{Performance comparison}

We first compare the computation times of the native schemes (mTSP, CovB, and ConB). Figure 1 shows the time required to generate the end-to-end paths for each scheme over $N_{m}$ (if all paths are assumed to be generated offline for fair comparison). The algorithms are implemented on an Intel Core i7-7820HQ processor at $2.90 \mathrm{GHz}$ (16 GB RAM). Due to the distributed nature and fewer operations, the computation time of CovB is the lowest. The computation time of mTSP depends on the number of cities (cells), number of iterations, and the population size, and does not change significantly with $N_{m}$. For ConB, on the other hand, $N_{m}$ has a direct impact on the depth-first-search phase of the connectivity check to the BS. The computation times of the hybrid schemes will also depend on $N_{m}$ due to the connectivity component and will approach ConB as $N_{m}$ increases and will be much higher than that of CovB. For smaller $N_{m}$, the computation time of mTSP-Con in comparison to mTSP will depend on the number of reserved search nodes $\left(N_{r}\right)$.

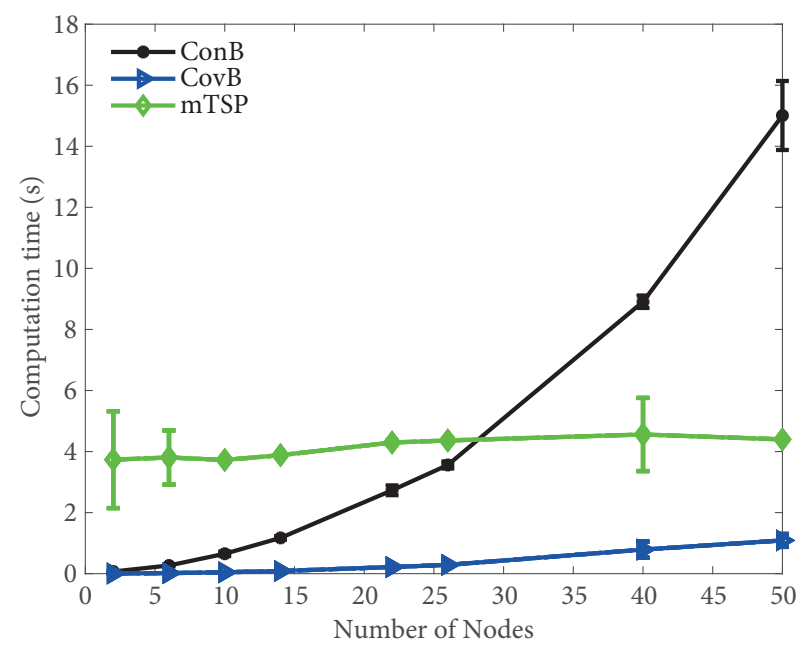

Figure 1. Path plan computation times versus number of nodes, when $r_{c}=500 \mathrm{~m}$.

Next, we study the impact of target duration and number of drones on the probability of detecting the target and informing the BS within $t_{d}$. Figure 2 shows the probability of success versus target duration $t_{d}$, when $N_{m}=\{10,22\}$, whereas Figure 3 shows the probability of success versus number of drones for fixed $t_{d}$. For this area size, a transmission range of $r_{c} \approx 2800 \mathrm{~m}$ would guarantee single-hop connectivity to all drones. Observe from the figures that as $r_{c}$ becomes larger mission success probability approaches 1 even with short target durations. In the case of ConB, the drones are allowed to spread more with higher transmission ranges. In the case of mTSP and CovB, with smaller $r_{c}$ the drones can inform the BS mostly on their return path. When $t_{d}=4000 \mathrm{~s}$, the mTSP scheme can finish the search mission even with 2 drones, and hence the mission success probability of mTSP in this case is 1 . Due to the constraints on connectivity, adding more drones does not help ConB, whereas higher spatial density (due to higher $r_{c}$ and/or $N_{m}$ ) improves the performance of mTSP and coverage-based schemes, significantly. The hybrid schemes highly benefit from integrating connectivity in the search decisions. The performance improvement of both schemes over ConB is most evident when $r_{c}$ is low. Between ConCov and mTSP-Con schemes, we observe that as $N_{m}$ is increased ConCov significantly outperforms mTSP-Con, implying that while mTSP-Con guarantees search time it still suffers from delayed notification. Due to the equal weight given to the search and connectivity components of ConCov, the drones 
can simultaneously spread out and stay connected. These results imply that while hybrid schemes utilize the available resources better than ConB, they might still require more resources than mTSP and CovB, especially when the detection task dominates the mission times.

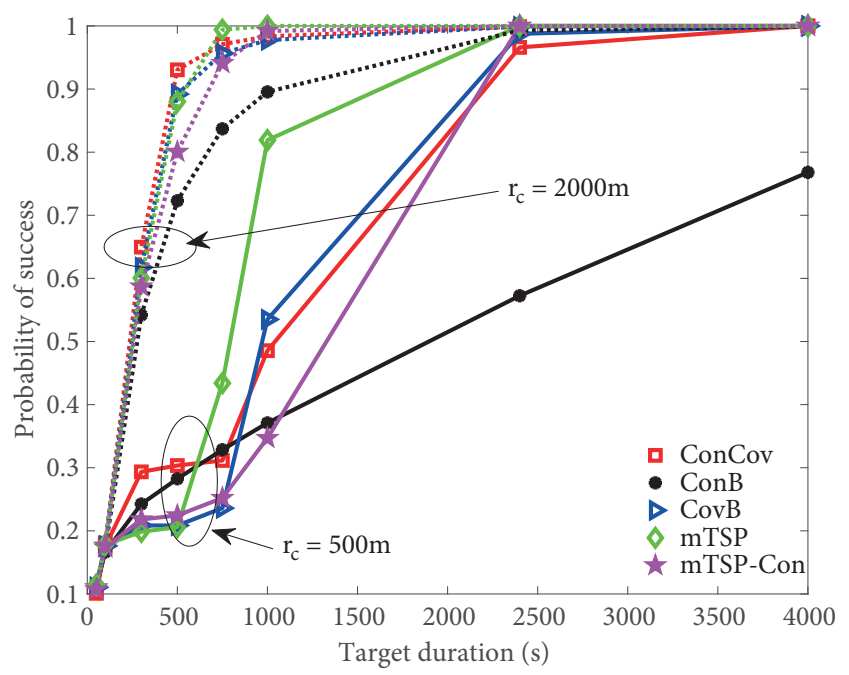

(a) $\mathrm{N}_{\mathrm{m}}=10$

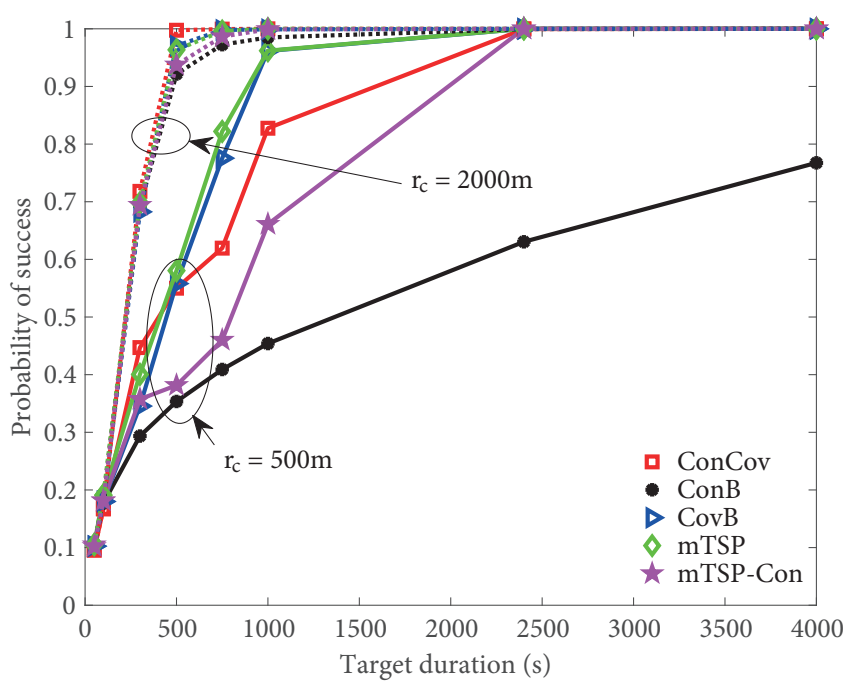

(b) $\mathrm{N}_{\mathrm{m}}=22$

Figure 2. Probability that the target information is delivered to the BS within $t_{d}$ versus target duration.

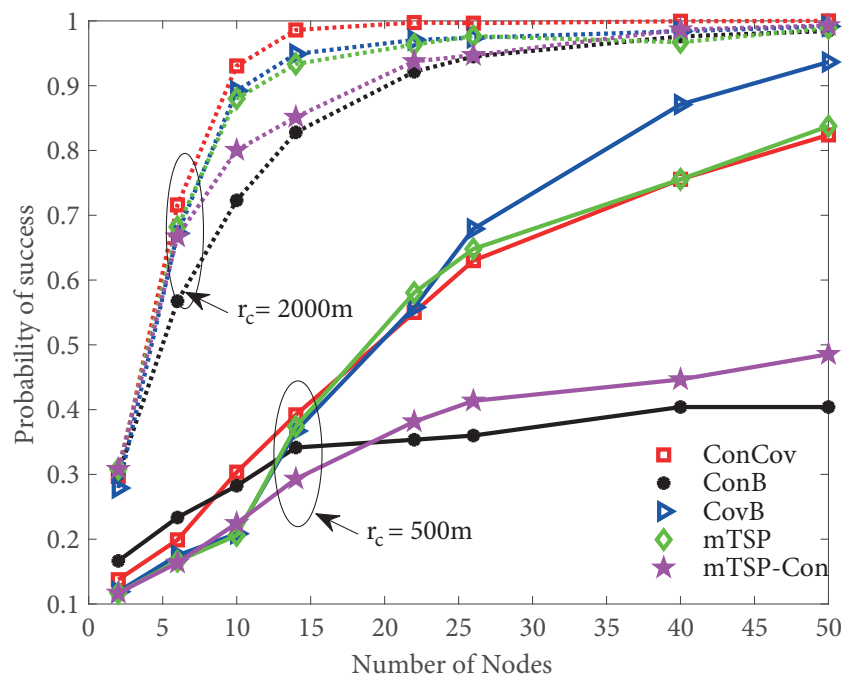

(a) $t_{d}=500 \mathrm{~s}$

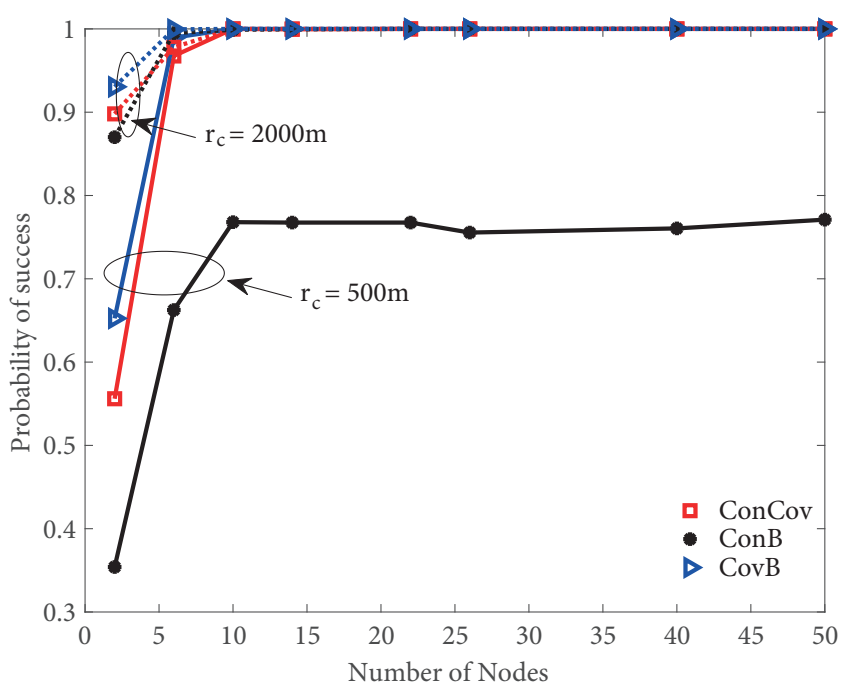

(b) $t_{d}=4000 s$

Figure 3. Probability that the target information is delivered to the BS within $t_{d}$ versus number of nodes.

Figure 4 shows the total mission time and the ratio of time spent for the search when $t_{d}=4000 \mathrm{~s}$. Figure $4 \mathrm{a}$ shows that as $N_{m}$ increases the performance of all schemes converge. However, the mission time of ConB is less than half of that of the other schemes for small $N_{m}$. While mTSP and CovB schemes aim 
to optimize search time, they suffer from lack of coordinated connectivity. Even when connectivity is added to the decision, total mission time is still higher for ConCov and mTSP-Con due to the time spent searching. This can be better observed in Figure $4 \mathrm{~b}$. For small $N_{m}$, the search time is dominant due to low resources. For higher $N_{m}$, search time also dominates over notification time due to high level of connectivity and hence improved immediate notification times. However, in between, the time spent for notification phase is highly significant for the native coverage schemes, implying that the likelihood of having connectivity to the BS at the time of detection is very low. ConCov has better notification times (30\% of mission time compared to $45 \%$, when $r_{c}=1000 \mathrm{~m}$ ) however, still much lower than ConB (10\% of total mission time for higher $r_{c}$ ). There is clearly a trade-off between immediate notification and fast detection. Observe that while the total mission time decreases monotonically with increasing $N_{m}$, there exists a number of drones that minimize the detection phase duration of the mission. While omitted here, similar trends have been observed for shorter $t_{d}$ as well. Therefore, depending on the priority of connectivity and tolerance to delays in notification, the choice of best path planning scheme changes.

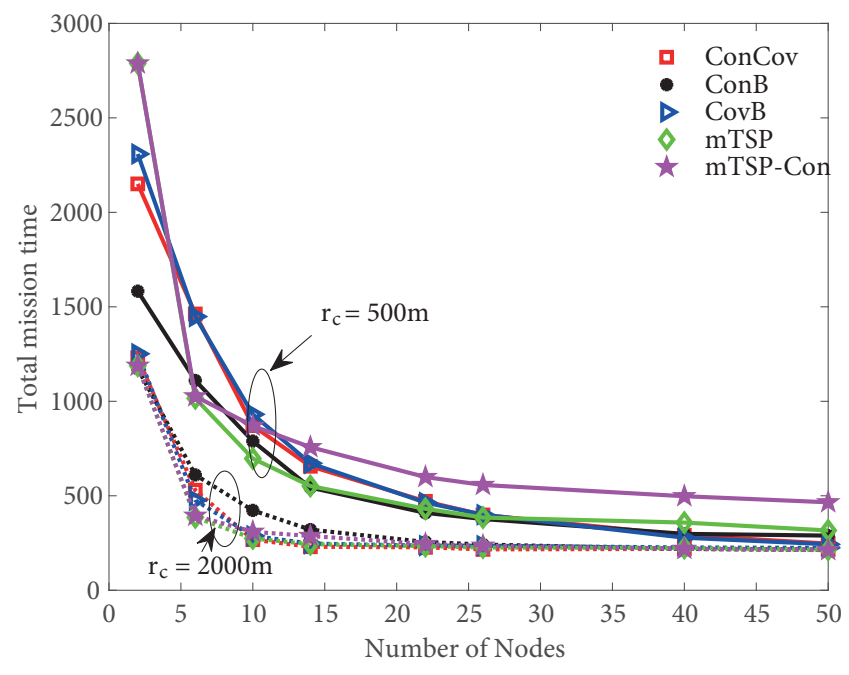

(a) Total mission time

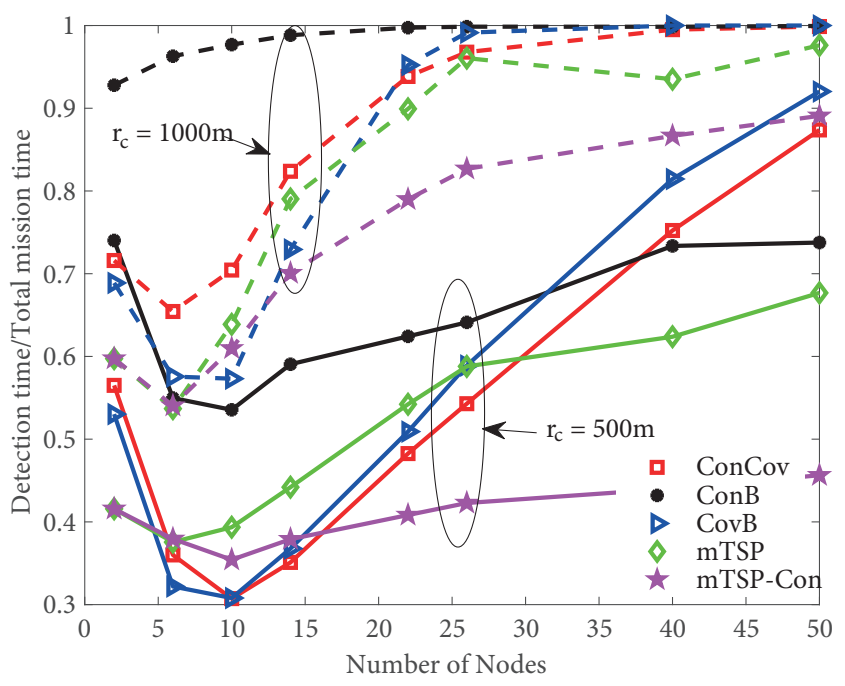

(b) Ratio of detection time over total mission time

Figure 4. Mission times versus number of nodes when $t_{d}=4000 \mathrm{~s}$.

We show in Figure 5 the probability of simultaneous detection and BS notification versus $N_{m}$. For short $t_{d}$, since the target is detected only if it is closer to the BS, immediate notification is highly likely. However, as $t_{d}$ increases, targets that are located farther from the BS can also be detected and the issue of notification becomes more critical. Therefore, we choose $t_{d}=4000 \mathrm{~s}$ to give the drones a chance to spread. For high $r_{c}$ the performance of all schemes converges to 1 ; hence these results are omitted. Observe that ConB, while suffering from lower detection probabilities, is much more likely to inform the BS at the time of detection. ConCov follows ConB. For lower $r_{c}$, the immediate notification probability of mTSP is the lowest (around 0.2), indicating that the BS is most likely notified on the return paths of drones. If some notification delay can be tolerated, mTSP preserves its advantage of minimum traveled distances in terms of mission success.

Finally, we compare the target reachability of the path planners versus $N_{m}$, when $t_{d}=4000 \mathrm{~s}$. It is computed by determining the percentage of target duration the target can be connected to the BS over a multihop link. Observe from Figure 6 that for small $r_{c}$ all schemes have low target reachability as expected. 
The ConB scheme outperforms the others in this case. As $r_{c}$ is increased, we observe that ConCov and CovB's performance starts exceeding that of $\mathrm{ConB}$ as $N_{m}$ increases, since ConB still suffers from lower detection probability. The mTSP scheme is the worst of all schemes even with higher $r_{c}$. Therefore, even if for coverage applications mTSP might be a good fit, for applications that might require continuous connectivity, such as surveillance, it suffers from lack of connectivity in the path decisions.

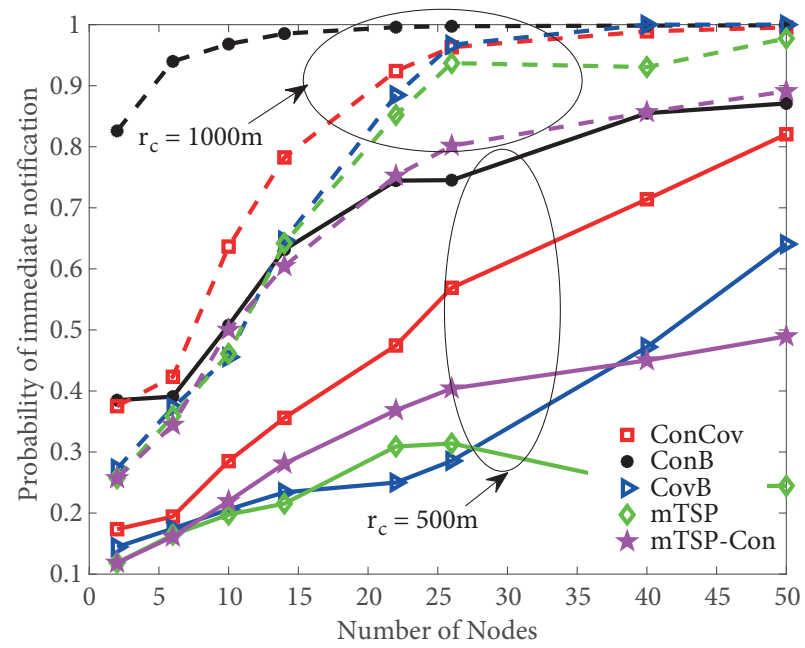

Figure 5. Probability of simultaneous detection and notification versus number of nodes when $t_{d}=4000 \mathrm{~s}$.

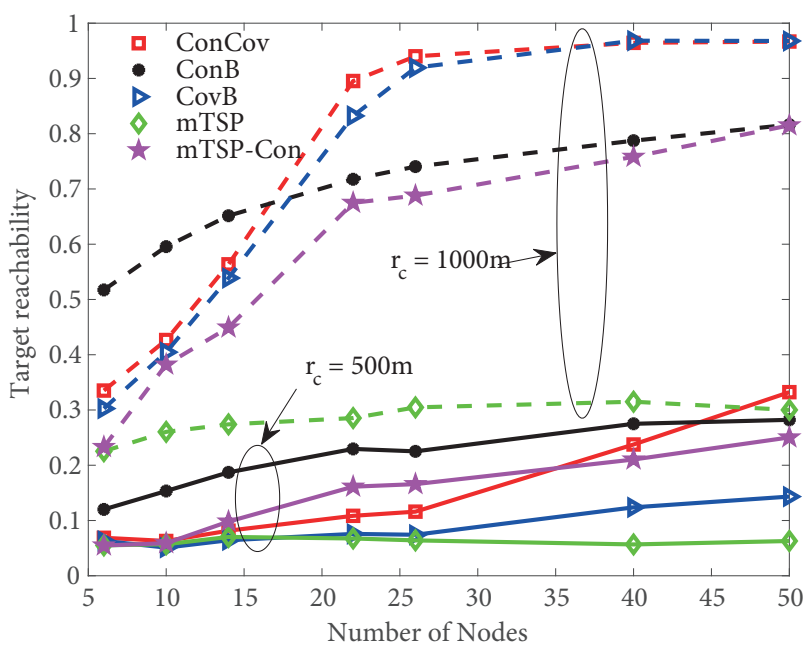

Figure 6. Percentage of time the target is connected to BS over a multihop link versus $N_{m}$ when $t_{d}=4000 \mathrm{~s}$.

\subsection{Impact of nonideal sensing and channel conditions}

In this section, our aim is to briefly illustrate how the performance trends are affected when the simplified sensing and connectivity assumptions are relaxed toward more realistic models. Figure 7a shows the probability of detection versus probability of missing a target, when $N_{m}=10, r_{c}=500 \mathrm{~m}$, and $t_{d}=1000 \mathrm{~s}$. In this scenario, we relax the error-free sensing assumption and assume that when the target is within the sensing coverage of any UAV, there is a probability that the target will not be detected. This implies that the target location will need to be sensed multiple times to detect the target with a high probability. The connectivity of the network is not affected by the sensing errors. Observe from Figure 7a that under ideal conditions when $t_{d}=1000 \mathrm{~s}$, coverage-based and hybrid schemes (mTSP, CovB,mTSP-Con, ConCov) are able to detect the target with probability higher than $90 \%$ (can also be seen in Figure 2a). When there are sensor errors, the detection probability decreases as expected. As was shown also previously [24], the detection probability is not significantly affected if the miss probability is not too high. Further observe that performance trends are changing in a similar manner for all schemes. This means the path planners can tolerate some errors if there is enough redundancy (i.e. sufficient number of UAVs and/or longer target duration) and under these conditions the findings from ideal conditions could be generalized. Further analysis is required to determine the robustness of the path planners against sensing errors and is outside the scope of the present paper; however, interested readers are referred to Khan et al. [18] for analysis of methods that utilize repetitive sensing and information sharing to overcome sensing imperfections.

Next, we analyze the impact of a more realistic channel model on the BS notification performance of the UAV team. In Section 4.2, we have assumed that the communication coverage area is a disc (i.e. two nodes 
are connected if they are within $r_{c}$ of each other). The communication range corresponded to the distancedependent path loss model. In the following, we change the channel to log-normal shadowing, which corresponds to large-scale fading in urban areas and includes fluctuations around the distance-dependent path loss due to multipath fading. While there are many other channel models, we adopt log-normal shadowing since it has been shown to fit the case where the UAV is flying over grass lawns and among buildings [17], which is suitable for an urban search and rescue scenario. We take the path-loss parameters from the air-ground link measurement campaign in Yang et al. [17]; i.e. the path loss exponent, $n$, is 1.75 and the standard deviation, $\sigma$, due to shadowing is $3 \mathrm{~dB}$. The corresponding path loss at a distance $(d)$ is given by

$$
P L(d)=P L_{0}+10 n \log _{10}\left(\frac{d}{d_{0}}\right)+S,
$$

where $P L_{0}$ is the path loss at a reference distance $d_{0}=1 \mathrm{~m}$ and $S$ is the shadow fading in log-normal distribution with $N\left(0, \sigma^{2}\right)$. Figure $7 \mathrm{~b}$ shows the probability of immediate notification versus $N_{m}$, when $r_{c}=1000 \mathrm{~m}$ and $t_{d}=4000 \mathrm{~s}$ (for comparison see Figure 5). Observe that the performance trends are similar to those of the simplified communication model, whereas the notification probability is improved slightly for mTSP schemes. Further analysis and preferably real-world tests are required to determine the real-time networking performance of the UAV team.

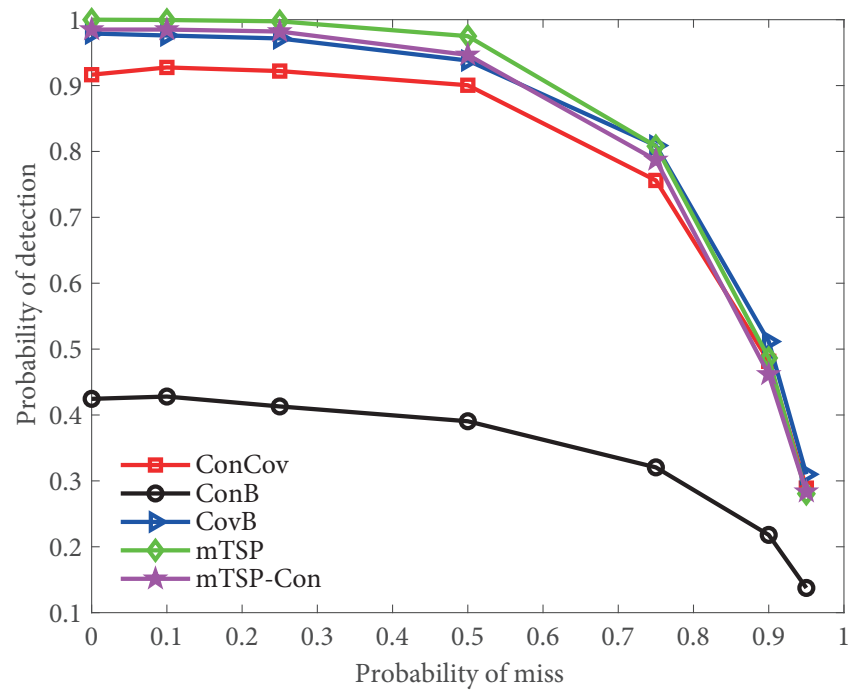

(a) Impact of imperfect sensing

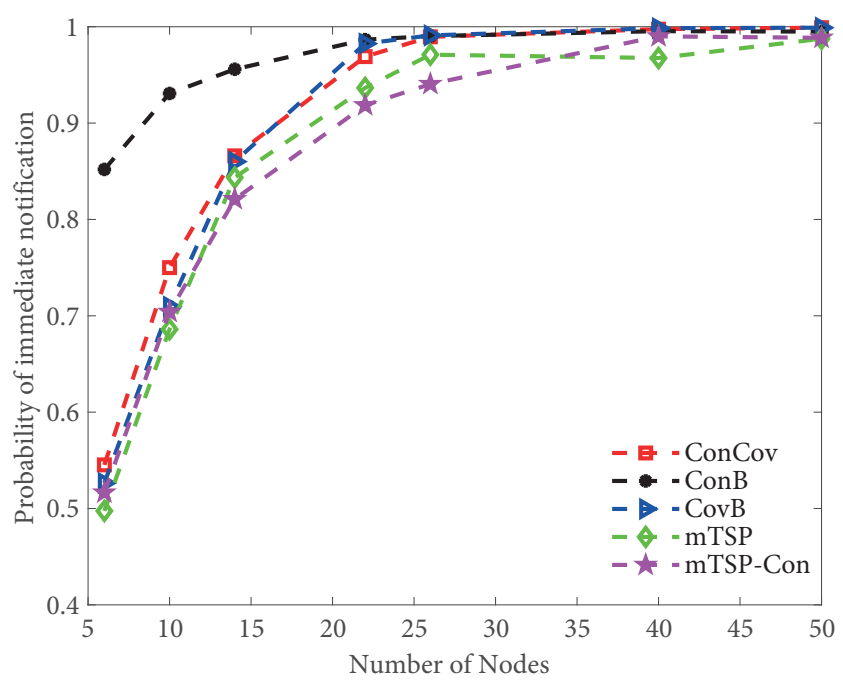

(b) Impact oflog-normal shadowing

Figure 7. (a) Probability of detection versus probability of target miss, when $N_{m}=10, r_{c}=500 \mathrm{~m}$, and $t_{d}=1000 \mathrm{~s}$; (b) Probability of immediate notification versus $N_{m}$, when $r_{c}=1000 \mathrm{~m}$ and $t_{d}=4000 \mathrm{~s}$.

\section{Conclusions}

In this work, we have analyzed centralized and distributed multi-UAV path planners for a search and rescue mission, where information about a detected target needs to be propagated to the ground BS. We have proposed two novel multi-UAV path planners that integrate connectivity limitations into coverage (search) path-planning algorithms. We have compared the performance of the proposed and benchmark planners in terms of mission success and mission times for ideal and nonideal sensing and channel conditions. 
YANMAZ ADAM/Turk J Elec Eng \& Comp Sci

Our results indicate that the impact of connectivity on the mission success probability and total mission times is most evident when communication resources (i.e. range and spatial density) are highly stringent. Therefore, the suitability of a given path-planning strategy for a given drone mission highly depends on the network connectivity requirements. While connectivity-aware path planning can provide simultaneous detection and notification as well as good target reachability, it suffers from longer search times due to slower spreading than the coverage-based schemes. On the other hand, mTSP schemes can find the targets fast, but require more tolerance to notification delays. Therefore, for a given application, the most suitable path planner and required resources will change. Our future work will focus on determining the optimum allocation of resources to different tasks of the mission.

\section{References}

[1] Yanmaz E, Yahyanejad S, Rinner B, Hellwagner H, Bettstetter C. Drone networks: communications, coordination, and sensing. Ad Hoc Networks 2018; 68 (1): 1-15.

[2] Hayat S, Yanmaz E, Muzaffar R. Survey on unmanned aerial vehicle networks for civil applications: a communications viewpoint. IEEE Communications Surveys \& Tutorials 2016; 18 (4): 2624-2661.

[3] Wu Q, Zeng Y, Zhang R. Joint trajectory and communication design for multi-UAV enabled wireless networks. IEEE Transactions on Wireless Communications 2018; 17 (3): 2109-2121.

[4] Waharte S, Trigoni N. Supporting search and rescue operations with UAVs. In: International Conference on Emerging Security Technologies (EST); Canterbury, UK. New York, NY, USA; 2010. pp. 142-147.

[5] Bourgault F, Furukawa T, Durrant-Whyte HF. Coordinated decentralized search for a lost target in a Bayesian world. In: International Conference on Intelligent Robots and Systems; Las Vegas, NV, USA; 2003. pp. $48-53$.

[6] Messous M, Senouci S, Sedjelmaci H. Network connectivity and area coverage for UAV fleet mobility model with energy constraint. In: IEEE Wireless Communications and Networking Conference; Doha, Qatar; 2016. pp. 1-6.

[7] Schleich J, Panchapakesan A, Danoy G, Bouvry P. UAV fleet area coverage with network connectivity constraint. In: 11th ACM International Symposium on Mobility Management and Wireless Access (MobiWac '13); New York, NY, USA; 2013. pp. 131-138.

[8] Trotta A, Felice MD, Montori F, Chowdhury KR, Bononi L. Joint coverage, connectivity, and charging strategies for distributed UAV networks. IEEE Transactions on Robotics 2018; 34 (4): 883-900.

[9] Yanmaz E. Connectivity versus area coverage in unmanned aerial vehicle networks. In: IEEE International Conference on Communications; Ottawa, Canada; 2013. pp. 719-723.

[10] Aggarwal S, Kumar N. Path planning techniques for unmanned aerial vehicles: a review, solutions, and challenges. Computer Communications 2020; 149 (1): 270-299.

[11] Al-Turjman F, Zahmatkesh H, Al-Oqily I, Daboul R. Optimized unmanned aerial vehicles deployment for static and mobile targets' monitoring. Computer Communications 2020; 149 (1): 27-35.

[12] Mostofi Y. Communication-aware motion planning in fading environments. In: IEEE International Conference on Robotics and Automation; Pasadena, CA, USA; 2008. pp. 3169-3174.

[13] Flushing EF, Kudelski M, Gambardella LM, Di Caro GA. Connectivity-aware planning of search and rescue missions. In: IEEE 2013 International Symposium on Safety, Security, and Rescue Robotics (SSRR); Linköping, Sweden; 2013. pp. $1-8$.

[14] Hayat S, Yanmaz E, Brown TX, Bettstetter C. Multi-objective UAV path planning for search and rescue. In: IEEE International Conference on Robotics and Automation; Singapore; 2017. pp. 5569-5574.

[15] Mozaffari M, Saad W, Bennis M, Debbah M. Efficient deployment of multiple unmanned aerial vehicles for optimal wireless coverage. IEEE Communications Letters 2016; 20 (8): 1647-1650. 
YANMAZ ADAM/Turk J Elec Eng \& Comp Sci

[16] Khuwaja AA, Chen Y, Zhao N, Alouini M, Dobbins P. A survey of channel modeling for UAV communications. IEEE Communications Surveys \& Tutorials 2018; 20 (4): 2804-2821.

[17] Yang Z, Zhou L, Zhao G, Zhou S. Channel model in urban environment for unmanned aerial vehicle communications. ArXiv, abs/1805.04328; 2018.

[18] Khan A, Yanmaz E, Rinner B. Information exchange and decision making in micro aerial vehicle networks for cooperative search. IEEE Transactions on Control of Network Systems, 2015; 2 (4): 335-347.

[19] Yanmaz E, Costanzo C, Bettstetter C, Elmenreich W. A discrete stochastic process for coverage analysis of autonomous UAV networks. In: 2010 IEEE Globecom Workshops (WiUAV); Miami, FL, USA; 2010. pp. 17771782.

[20] Bektas T. The multiple traveling salesman problem: an overview of formulations and solution procedures. Omega 2006; 34 (3): 209-219.

[21] Potvin JY. Genetic algorithms for the traveling salesman problem. Annals of Operations Research 1996; 63 (3): 337-370.

[22] Yanmaz E, Guclu H. Stationary and mobile target detection using mobile wireless sensor networks. In: 2010 INFOCOM IEEE Conference on Computer Communications Workshops; San Diego, CA, USA; 2010. pp. 1-5.

[23] Yanmaz E. Leveraging connectivity for coverage in drone networks for target detection. Balkan Journal of Electrical and Computer Engineering 2019; 7 (3): 218-225. doi:10.17694/bajece.503818

[24] Yanmaz E, Bettstetter C. Area coverage with unmanned vehicles: a belief-based approach. In: IEEE Vehicular Technology Conference (VTC); Taipei, Taiwan; 2010. pp. 1-5. 